\title{
Long non-coding RNA HOTAIR promotes cell viability, migration and invasion in thyroid cancer by sponging miR-17-5p
}

\author{
X. LIU, G. LIU, Y. LU, Y. SHI ${ }^{*}$ \\ Department of Endocrinology, Tangshan Workers Hospital, Tangshan, China \\ *Correspondence: tsdbyylxl2011@163.com
}

Received March 20, 2019 / Accepted May 22, 2019

\begin{abstract}
Thyroid cancer is a common endocrine malignancy. Long non-coding RNAs (lncRNAs) were reported to regulate cellular processes in tumorigenesis and development. In this study, we mainly investigated the role of Homeobox Transcript Antisense Intergenic RNA (HOTAIR) in thyroid cancer and its potential mechanism. RT-qPCR was conducted to evaluate the expression of HOTAIR and miR-17-5p in thyroid cancer tissues and 4 cell lines (TPC-1, FTC-133, B-CPAP and SW579). MTT assay was employed to analyze the cell viability of TPC-1 and FTC-133 cells. The migration and invasion of TPC-1 and FTC-133 cells were detected via Transwell assay. Luciferase reporter assay was used to validate the target of HOTAIR. In vivo tumor formation assay in nude mice was applied to investigate the impact of HOTAIR on thyroid cancer tumorigenesis. HOTAIR was significantly upregulated, while miR-17-5p was downregulated in thyroid cancer tissues and cells, and the miR-17-5p expression in thyroid cancer tissues was inversely correlated with HOTAIR expression. Silencing HOTAIR significantly inhibited the cell viability, migration and invasion of TPC-1 and FTC-133 cells. MiR-17-5p was a target of HOTAIR and counter-regulated by HOTAIR. Introduction of miR-17-5p also inhibited the cell viability, migration and invasion in TPC-1 and FTC-133 cells. Moreover, introduction of miR-17-5p reversed knockdown of HOTAIR-mediated the suppression effects on the cell viability, migration and invasion in thyroid cancer cells. Additionally, knockdown of HOTAIR inhibited thyroid cancer tumorigenesis in mice. Our results suggested HOTAIR promotes cell viability, migration and invasion in thyroid cancer cells by sponging miR-17-5p.
\end{abstract}

Key words: HOTAIR, thyroid cancer, cell viability, migration and invasion, miR-17-5p

Thyroid cancer is the fifth most common cancer in women in the USA and is more prevalent in women and elderly individuals $[1,2]$. In 2015, an estimated over 62, 000 new cases of thyroid cancer incidence occurred in the USA [3]. Despite great advancements in the prognosis and treatment of thyroid cancer, $10-20 \%$ of thyroid cancer patients develop chemotherapeutic resistance and undergo recurrence [4]. So, there is an urgent need for investigation on thyroid cancer progression as thorough as possible.

Long non-coding RNAs (lncRNAs) are a class of RNAs with transcripts $>200 \mathrm{nt}$ in length and certain lncRNAs are now proven to play important roles in critical cellular processes [5]. Former studies have indicated that lncRNAs could sponge miRNA to modulate gene expression and participate in the cellular processes [6]. HOTAIR is linked with the development or progression of spermiogenesis, aortic valve calcification and neurodegeneration diseases, as well as participates in regulating osteogenic differentiation and proliferation through modulating miR-17-5p and its target gene SMAD7 [7]. HOTAIR has been confirmed to have an extremely important role in oncogenic progression of colorectal cancer, and HOTAIR blood levels might serve as a potential prognostic marker in sporadic CRC [8]. LncRNA HOTAIR is involved in papillary thyroid carcinoma (PTC) development and plays oncogenic role [9]. HOTAIR expression in thyroid cancer is probably relevant to the aggressiveness and the progression of the tumor, and HOTAIR could be a promising biomarker in patients with thyroid cancer [10].

Similar to lncRNAs, microRNAs (miRNAs) are non-coding RNAs. They are endogenous, small RNAs (19-25 nucleotides in length) that are involved in cancer initiation and progression $[11,12]$. Known as key negative regulators of targeted gene expression, miRNAs regulate gene expression by acting on their pairing with messenger RNAs (mRNAs), contributing to their degradation inhibiting translation [13]. Certain miRNAs could be used to develop, refine or strengthen strategies for diagnosis and management of 
thyroid cancer, serving as biomarker in thyroid cancer [14]. MiR-17-5p is a member of the miR-17-92 cluster, located on chromosome 13q31-32, which exerts critical function in regulation on multiple related cellular processes, like proliferation and differentiation, during development and disease $[15,16]$. An increasing studies revealed that miR-17-5p has dual function in tumor progression [17].

In the present study, we analyzed the expression levels of HOTAIR and miR-17-5p in thyroid cancer tissues and cell lines, the functional role of HOTAIR and miR-17-5p in thyroid cancer progression in vitro and in vivo. We also explored the potential regulatory mechanism.

\section{Materials and methods}

Clinical samples and cell culture. The 30 pairs of thyroid cancer tissues and corresponding adjacent normal thyroid tissues were collected from 30 patients diagnosed as thyroid cancer at Tangshan Workers Hospital from May 2016 to August 2018. Our study was approved by the Research Ethics Committee of Tangshan Workers Hospital. All patients signed informed consents. Samples were immediately frozen in liquid nitrogen and stored at $-80^{\circ} \mathrm{C}$ until used.

Human thyroid follicular cell line Nthy-ori3-1 was purchased from Shanghai Institute of Cell Biology of the Chinese Academy of Sciences (Shanghai, China), and human thyroid cancer cell lines TPC-1, FTC-133, B-CPAP and SW579 were purchased from American Type Culture Collection (ATCC, Manassas, USA). Nthy-ori3-1 cells were maintained in Roswell Park Memorial Institute 1640 Medium (RPMI1640; Gibco, Grand Island, USA) supplemented with $10 \%$ fetal bovine serum (FBS; Thermo Fisher Scientific, USA) and $100 \mathrm{U} / \mathrm{ml}$ penicillin $\mathrm{G}$ sodium salt and $100 \mathrm{U} / \mathrm{ml}$ streptomycin sulfate. TPC-1, FTC-133, B-CPAP and SW579 cells were maintained in Dulbecco's Modified Eagle Medium (DMEM, HyClone, Logan, USA). All the cell lines were cultured in a $5 \% \mathrm{CO}_{2}$ humidified incubator at $37^{\circ} \mathrm{C}$.

Reagent and cell transfection. The si-HOTAIR and its negative control si-NC, AgomiR-17-5p and its negative control AgomiR-NC, AntagomiR-17-5p and its negative control AntagomiR-NC, were synthesized by GenePharma Co. Ltd. (Shanghai, China). Above mentioned plasmids or oligonucleotides were transfected into TPC-1 and FTC-133 cells using Lipofectamine ${ }^{\mathrm{TM}} 2000$ reagent (Invitrogen, Carlsbad, USA) following the protocols supplied by the manufacturer. At $48 \mathrm{~h}$ post-transfection, cells were collected for further assay.

Reverse transcription-quantitative polymerase chain reaction (RT-qPCR) assay. Total cellular RNA was isolated from tissues or cells using the utilizing TRIzol reagent (Life Technologies, Grand Island, USA). PrimeScript RT Reagent kit (Takara Bio, Inc., Otsu, Japan) was used to convert 500 ng RNA into cDNA. For mRNA expression detection, qPCR was conducted using Power SYBR Green Master Mix (Applied Biosystems, Foster City, USA).
For miRNA expression detection, qPCR was conducted with miRNA-specific TaqMan MiRNA Assay Kit (Applied Biosystems). qPCR was performed on CFX96 ${ }^{\text {Th }}$ Real-time PCR Detection System (Bio-Rad Laboratories, Hercules, USA). GAPDH and U6 were used as the internal control for HOTAIR and miR-17-5p, respectively. The primers for HOTAIR, GAPDH, miR-17-5p and U6 were listed as below: HOTAIR, 5'-TTTGGACTGTAAAATATGGC-3' (forward) and 5'-TTCTGACACTGAACGGACT-3' (reverse); GAPDH, 5'-ACCTGACCTGCCGTCTAGAA-3 (forward) and 5'-TCCACCACCCTGTTGCTGTA-3' (reverse); miR-17-5p, 5'-CCAGGATCCTTTATAGTTGTTAGAGTTTG-3' (forward) and 5'-CGGAATTCTAATCTACTTCACTATCTGC-3' (reverse); U6, 5'-CTCGCTTCGGCAGCACA-3 (forward) and 5'-AACGCTTCACGAATTTGCGT-3' (reverse). The expression of HOTAIR and miR-17-5p were analyzed using the $2^{\Delta \mathrm{Ct}}$ method.

MTT assay. Transfected TPC- 1 and FTC- 133 cells (about $3 \times 10^{3}$ per well) were inoculated in 96-well plates and cultured at $37^{\circ} \mathrm{C}$ for $24 \mathrm{~h}, 48 \mathrm{~h}$ and $72 \mathrm{~h}$. With $20 \mu \mathrm{l}$ MTT solution (Sigma-Aldrich, St. Louis, USA) added to each well, the cells were incubated at $37^{\circ} \mathrm{C}$ for another $4 \mathrm{~h}$. The supernatant was removed, $150 \mu$ dimethyl sulfoxide (DMSO, Sigma-Aldrich) was added into each well. The absorbance at $570 \mathrm{~nm}$ was measured using a microplate reader (Infinite M200; Tecan Group, Ltd., Switzerland).

Migration and invasion assays. For migration assay, $3 \times 10^{4}$ transiently transfected TPC- 1 and FTC-133 cells were seeded in DMEM in the upper chamber $(8 \mu \mathrm{m}$ pores; BD Biosciences, San Jose, USA) respectively, while DMEM containing 10\% FBS was added to the lower chamber. At $48 \mathrm{~h}$ post-incubation, cells adhering to the bottom of the Transwell membrane were stained with $0.1 \%$ crystal violet at room temperature for $15 \mathrm{~min}$, then imaged using Olympus X-71 inverted light microscope (Olympus, Tokyo, Japan). Additionally, crystal violet in the stained cells derived from the lower chamber was dissolved in $10 \%$ acetic acid quantified by measuring the absorbance at $595 \mathrm{~nm}$ as previously described [18] using a microplate reader. The cell invasion assays were performed using similar protocol but instead, cells were seeded in the upper chamber pre-coated with Matrigel (BD Biosciences).

Luciferase reporter assay. The fragment from HOTAIR containing the putative binding site of miR-17-5p and its mutant were amplified by PCR and cloned into pGL3 luciferase promoter vector (pGL3-empty, Promega, Madison, USA) to synthesize WT-HOTAIR or MUT-HOTAIR. Then the TPC-1 and FTC-133 cells were co-transfected with AgomiR-17-5p and WT-HOTAIR or MUT-HOTAIR using Lipofectamine ${ }^{\mathrm{mw}} 2000$ reagent. After $48 \mathrm{~h}$, co-transfected cells were harvested, and the luciferase activity was examined using the Dual Luciferase Reporter Detection System (Promega).

Tumor formation in nude mice. This experiment was approved by Research Ethics Committee of Tangshan 
Workers Hospital. Transfected FTC- 133 cells $\left(1 \times 10^{7}\right.$ cells in $200 \mu \mathrm{l}$ DMEM without serum) were hypodermically injected into 5-week-old BALB/c male nude mice, obtained from Shanghai SLAC Laboratory Animal Co., Ltd. (Shanghai, China; $\mathrm{n}=6$ per group). Nude mice were maintained as previously described [17]. At specific time point, the mice were euthanized, and the volume and weight of the tumors were measured.

Statistical analysis. SPSS 22.0 software (SPSS, Chicago, IL, USA) were used for statistical analysis. The data were exhibited as mean \pm SD (standard deviation) as indicated. Student's t-test was conducted to assess statistical differences between two groups. A p-value less than 0.05 was considered statistically significant. All experiments were performed for three times.

\section{Results}

HOTAIR was upregulated in thyroid cancer tissues and cell lines. To verify the impact of HOTAIR on the progression of thyroid cancer, we firstly examined the HOTAIR expression level in thyroid cancer tissues and paired adjacent normal tissues via RT-qPCR. We found that HOTAIR was highly expressed in thyroid cancer tissues, compared with corresponding adjacent noncancerous tissues (Figure 1A). The HOTAIR expression was also detected in human thyroid follicular cell line Nthy-ori3-1 and 4 human thyroid cancer cell lines (TPC-1, FTC-133, B-CPAP and SW579). Obvious augment of HOTAIR expression was observed in all above mentioned thyroid cancer cell lines (Figure 1B).

Knockdown of HOTAIR inhibited cell viability, migration and invasion abilities in thyroid cancer cells in vitro. To clarify the function role of HOTAIR on thyroid cancer cells, si-HOTAIR was transiently transfected into TPC-1 and FTC-133 cells. As shown in Figure 2A, the TPC-1 and FTC-133 cells with HOTAIR downregulated were successfully constructed. MTT assay was applied to figure out the effect of HOTAIR on the cell viability of TPC-1 and FTC-133 cells and the results revealed that knockdown of HOTAIR effectively inhibited cell viability of TPC- 1 and FTC-133 cells, compared with cells transfected with si-NC (Figures 2B and $2 \mathrm{C})$. Transwell assay indicated that both the relative migration (Figures 2D and 2E) and invasion (Figures 2F and 2G) rate of TPC- 1 and FTC-133 cells transfected with si-HOTAIR were distinctly lower than that in cells transfected with si-NC (Figures 2D-2G).

MiR-17-5p was a direct target of HOTAIR and negatively regulated by HOTAIR. Dual-luciferase reporter assay was employed to further validate the target relationship between HOTAIR and miR-17-5p. Strikingly, the luciferase activity of TPC- 1 and FTC- 133 cells co-transfected with AgomiR-17-5p and WT-HOTAIR was significantly reduced, while the luciferase activity of cells co-transfected with AgomiR-17-5p and MUT-HOTAIR exhibited no apparent change (Figures 3A and $3 \mathrm{~B}$ ). To verify whether HOTAIR modulates miR-17-5p, RT-qPCR was carried out to detect the expression level of miR-17-5p in TPC-1 and FTC-133 cells. Results revealed that overexpression of HOTAIR reduced miR-17-5p expression while knockdown of HOTAIR enhanced miR-17-5p expression in the two cell lines (Figure 3C).

MiR-17-5p was significantly downregulated in thyroid cancer tissues and cell lines. To investigate the miR-17-5p expression in thyroid cancer tissues and cell lines, RT-qPCR assay was conducted. We observed that the expression level of miR-17-5p in thyroid cancer tissues was evidently lower than that in normal tissues (Figure 4A). Analogously, miR-17-5p was significantly downregulated in thyroid cancer TPC-1 and FTC-133 cells, compared to Nthy-ori3-1 cells

\section{A}

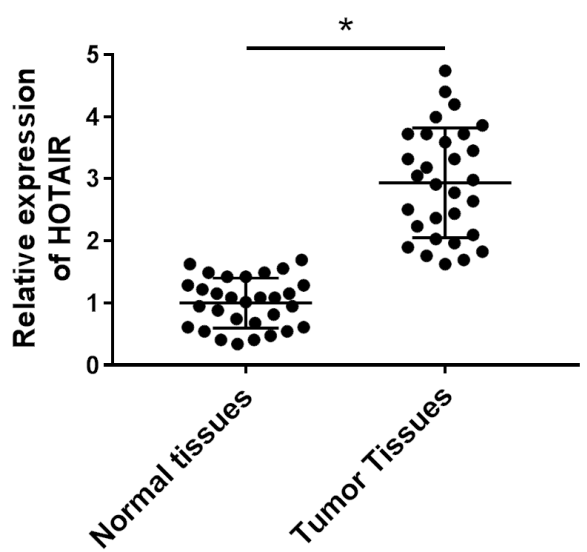

B

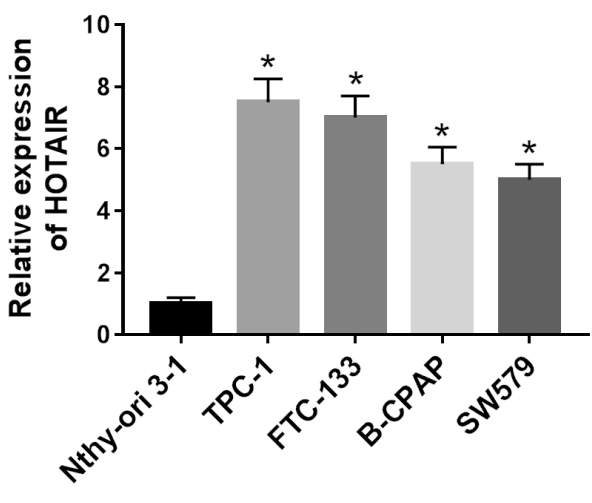

Figure 1. HOTAIR was upregulated in thyroid cancer tissues and cell lines. A) The expression level of HOTAIR in 30 pairs of thyroid cancer tissues and corresponding adjacent normal tissues was tested by RT-qPCR. ${ }^{*}$ p $<0.05$ compared to normal tissues. B) The HOTAIR expression of human thyroid follicular cell line Nthy-ori3-1 and 4 thyroid cancer cell lines (TPC-1, FTC-133, B-CPAP and SW579) was also detected by RT-qPCR. ${ }^{\star}$ p $<0.05$ compared to Nthy-ori3-1 cells. 


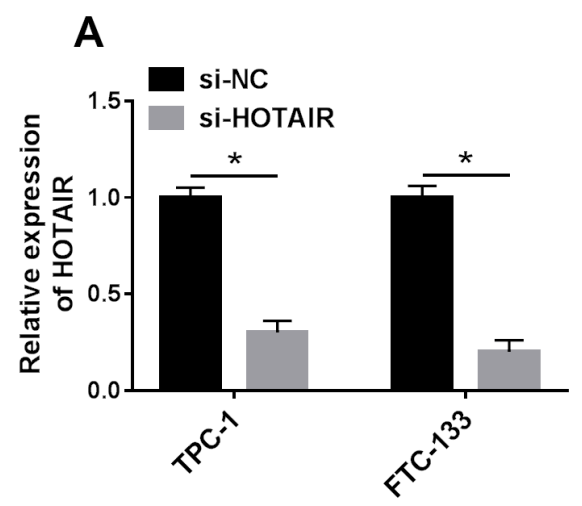

B

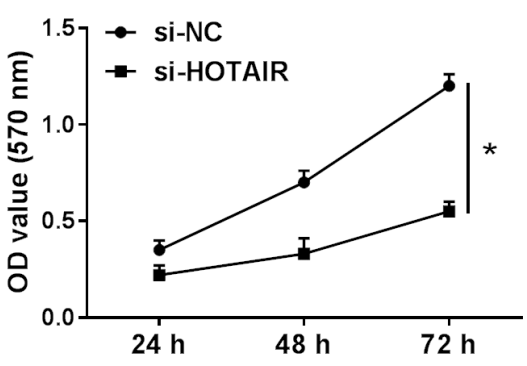

C

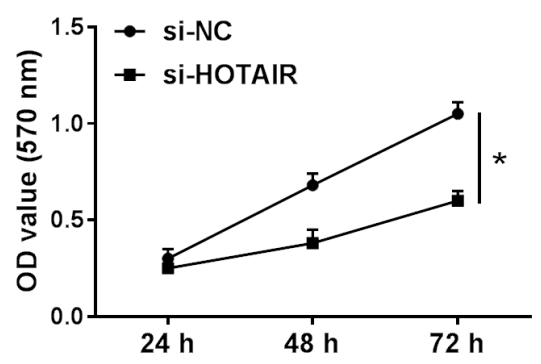

D

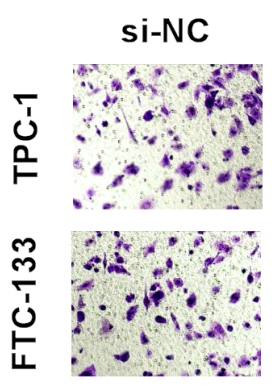

Si-HOTAIR

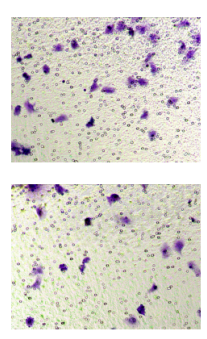

E

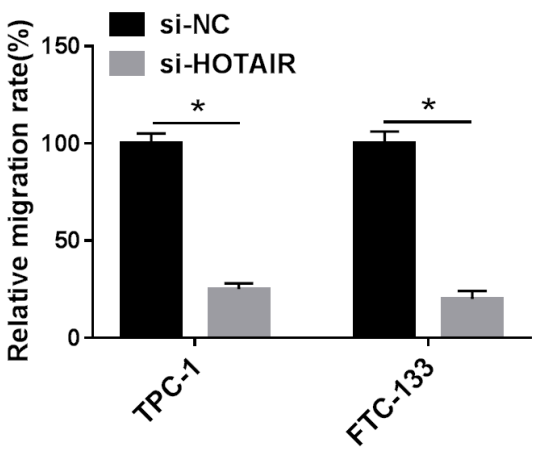

G

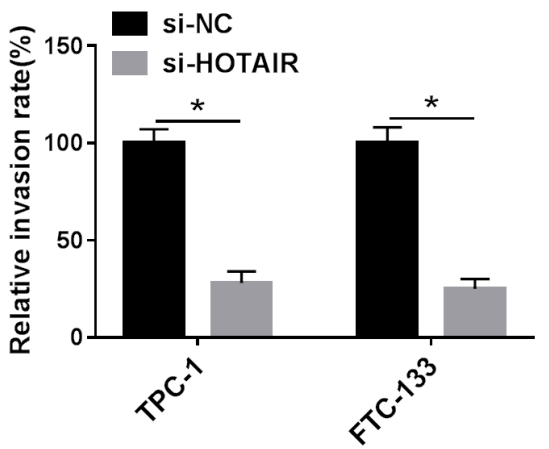

Figure 2. Knockdown of HOTAIR inhibited cell viability, migration and invasion abilities in thyroid cancer cells in vitro. TPC-1 and FTC-133 cells were transfected with si-HOTAIR or si-NC. A) RT-qPCR was conducted to evaluate the HOTAIR expression in TPC-1 and FTC-133 cells after transfection. $\mathrm{B}$ and C) MTT assay for TPC-1 and FTC-133 cells after transfection for $24 \mathrm{~h}, 48 \mathrm{~h}$ and $72 \mathrm{~h}$. D and E) The relative migration rate of TPC-1 and FTC133 cells after transfection conducted on Transwell. F and G) The relative invasion rate of TPC-1 and FTC-133 cells after transfection conducted on Transwell. ${ }^{\star} \mathrm{p}<0.05$ compared to cells transfected with si-NC.

(Figure 4B). Pearson analysis showed that the expression level of miR-17-5p in thyroid cancer tissues was inversely correlated with HOTAIR expression (Figure 4C).

Upregulation of miR-17-5p repressed the cell viability, migration and invasion abilities in thyroid cancer cells in vitro. To explore the effect of miR-17-5p on thyroid cancer cells, we transfected thyroid cancer TPC-1 and FTC-133 cells with AgomiR-17-5p to enhance miR-17-5p expression in the two cell lines (Figure 5A). MTT assay was employed to determine the influence of overexpression of miR-17-5p on the cell viability of TPC-1 and FTC-133 cells. The results suggested that overexpression of miR-17-5p repressed the cell viability of the two cell lines (Figures $5 \mathrm{~B}$ and $5 \mathrm{C}$ ). Transwell assay demonstrated that overexpression of miR-17-5p also hindered migration (Figure 5D) and invasion (Figure 5E) abilities of TPC-1 and FTC-133 cells (Figures 5D and 5E). 

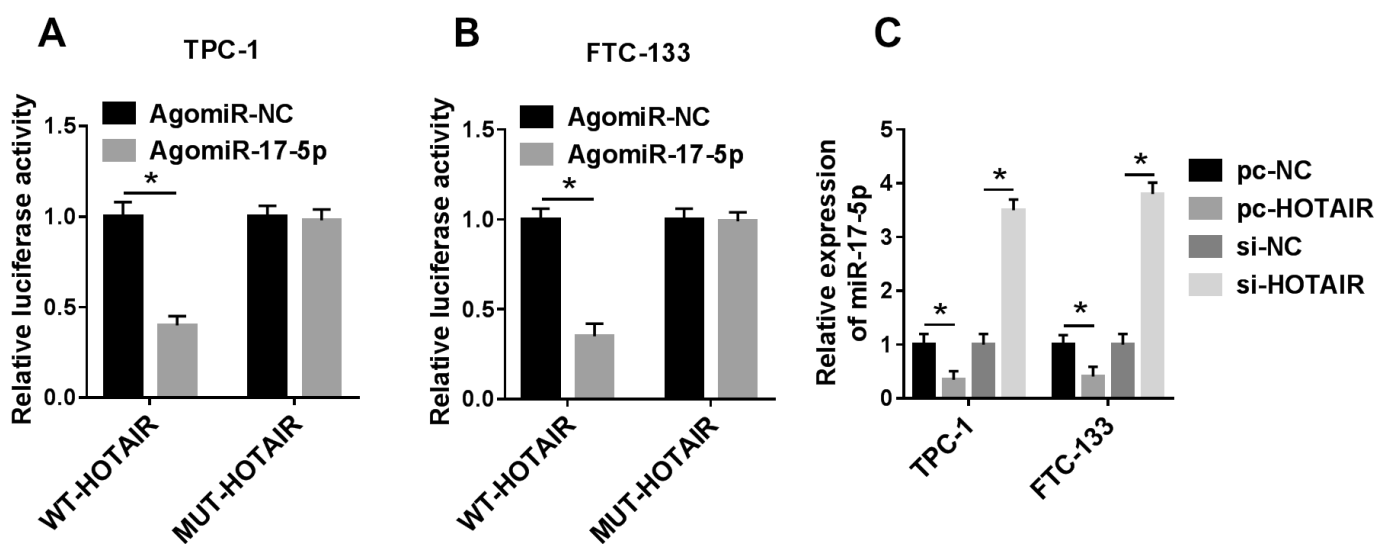

Figure 3. MiR-17-5p was a direct target of HOTAIR and negatively regulated by HOTAIR. A and B) Dual-luciferase reporter assays for TPC-1 and FTC133 cells co-transfected with WT-HOTAIR or MUT-HOTAIR plasmid and AgomiR-17-5p or AgomiR-NC, ${ }^{\star} \mathrm{p}<0.05$ compared to cells co-transfected with WT-HOTAIR and AgomiR-NC. C) The expression of miR-17-5p in TPC-1 and FTC-133 cells transfected with pc-HOTAIR, pc-NC, si-HOTAIR or si-NC. ${ }^{*} \mathrm{p}<0.05$ compared to cells transfected with pc-NC or si-NC.
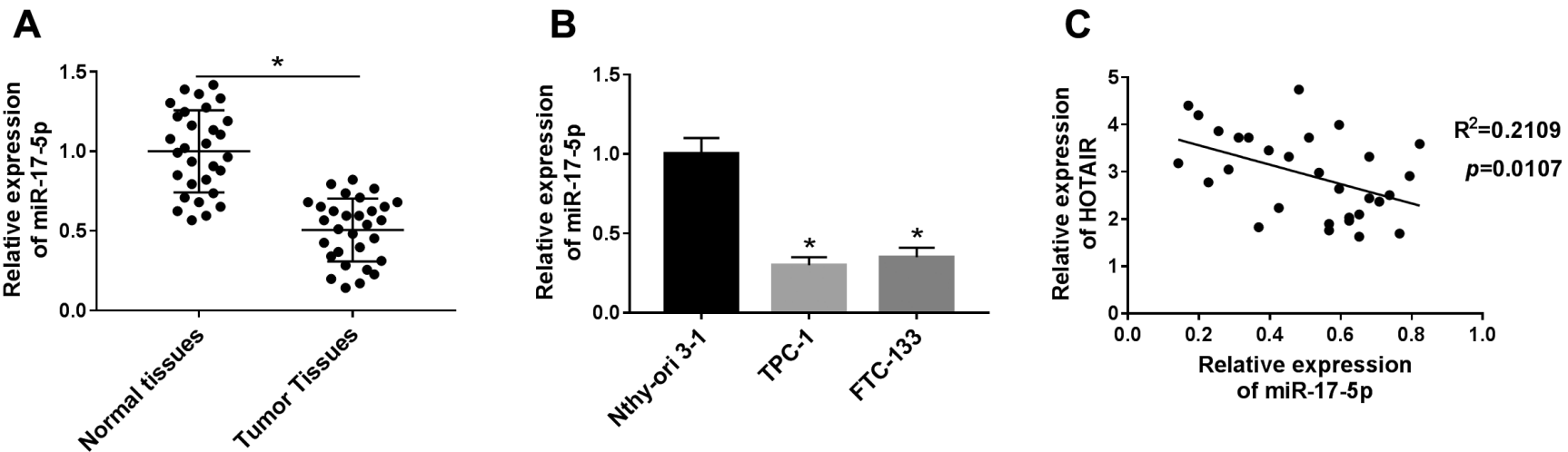

Figure 4. MiR-17-5p was significantly downregulated in thyroid cancer tissues and cell lines. A) The expression level of miR-17-5p in 30 pairs of thyroid cancer tissues and corresponding adjacent normal tissues was detected by RT-qPCR. ${ }^{*} p<0.05$ compared to normal tissues. B) The HOTAIR expression of human thyroid follicular cell line Nthy-ori3-1 as well as thyroid cancer TPC-1 and FTC-133 cells was also detected by RT-qPCR. ${ }^{\star}$ p $<0.05$ compared to Nthy-ori3-1 cells. C) Correlation analysis between miR-17-5p and HOTAIR in thyroid cancer tissues.

Downregulation of miR-17-5p reversed si-HOTAIRmediated inhibition on the cell viability, migration and invasion abilities in thyroid cancer cells. To figure out whether HOTAIR performed its function by targeting miR-17-5p in thyroid cancer cells, the rescue experiments were conducted. We first co-transfected si-HOTAIR and AntagomiR-17-5p into TPC-1 and FTC-133 cells, as shown in Figure 6A, AntagomiR-17-5p obviously restored miR-17-5p expression, which was enhanced by si-HOTAIR. MTT assay revealed that downregulation of HOTAIR blocked the cell viability of TPC- 1 and FTC-133 cells, while the effect could be mitigated by downregulation of miR-17-5p (Figures 6B and $6 \mathrm{C}$ ). As for the migration and invasion, silencing of HOTAIR decreased the migration and invasion abilities, whereas AntagomiR-17-5p effectively rescued the reduction of migration and invasion abilities of TPC-1 (Figure 6D) and FTC-133 (Figure 6E) cells stimulated by si-HOTAIR (Figures 6D and 6E).

Knockdown of HOTAIR inhibited thyroid cancer tumorigenesis in vivo. Additionally, we conducted in vivo experiment to investigate the effect of HOTAIR on thyroid cancer tumorigenesis. Tumors were collected from athymic mice injected with FTC-133 cells transfected with si-HOTAIR or si-NC. The volume of tumors from mice injected with FTC-133 cells transfected with si-HOTAIR was smaller than in si-NC group (Figure 7A). The tumor weight in si-HOTAIR group was also lower than in si-NC group (Figure 7B). RT-qPCR assay demonstrated that HOTAIR was downregulated (Figure 7C), while miR-17-5p showed the opposite tendency (Figure 7D) in mice injected with FTC-133 cells transfected with si-HOTAIR (Figures 7C and 7D). 


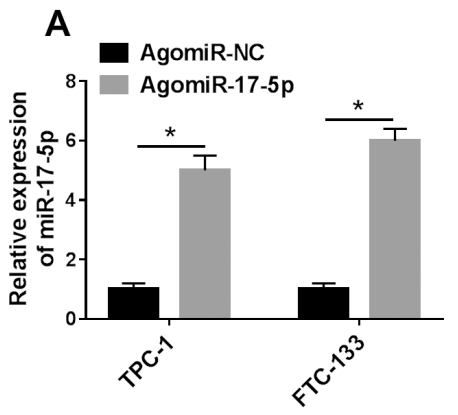

D

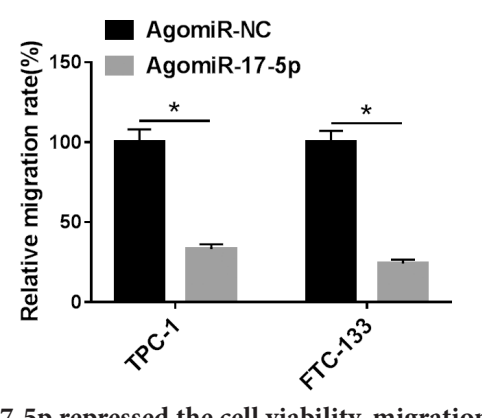

Figure 5. Upregulation of miR-17-5p repressed the cell viability, migration and invasion abilities in thyroid cancer cells in vitro. TPC-1 and FTC-133 cells were transfected with AgomiR-17-5p or AgomiR-NC. A) RT-qPCR was utilized to detect miR-17-5p expression in TPC-1 and FTC-133 cells after transfection. B and C) MTT assay for TPC-1 and FTC-133 cells after transfection for $24 \mathrm{~h}, 48 \mathrm{~h}$ and $72 \mathrm{~h}$. D) The relative migration rate of TPC-1 and FTC-133 cells after transfection conducted on Transwell. E) The relative invasion rate of TPC-1 and FTC-133 cells after transfection conducted on Transwell. ${ }^{\star} \mathrm{p}<0.05$ compared to cells transfected with AgomiR-NC.

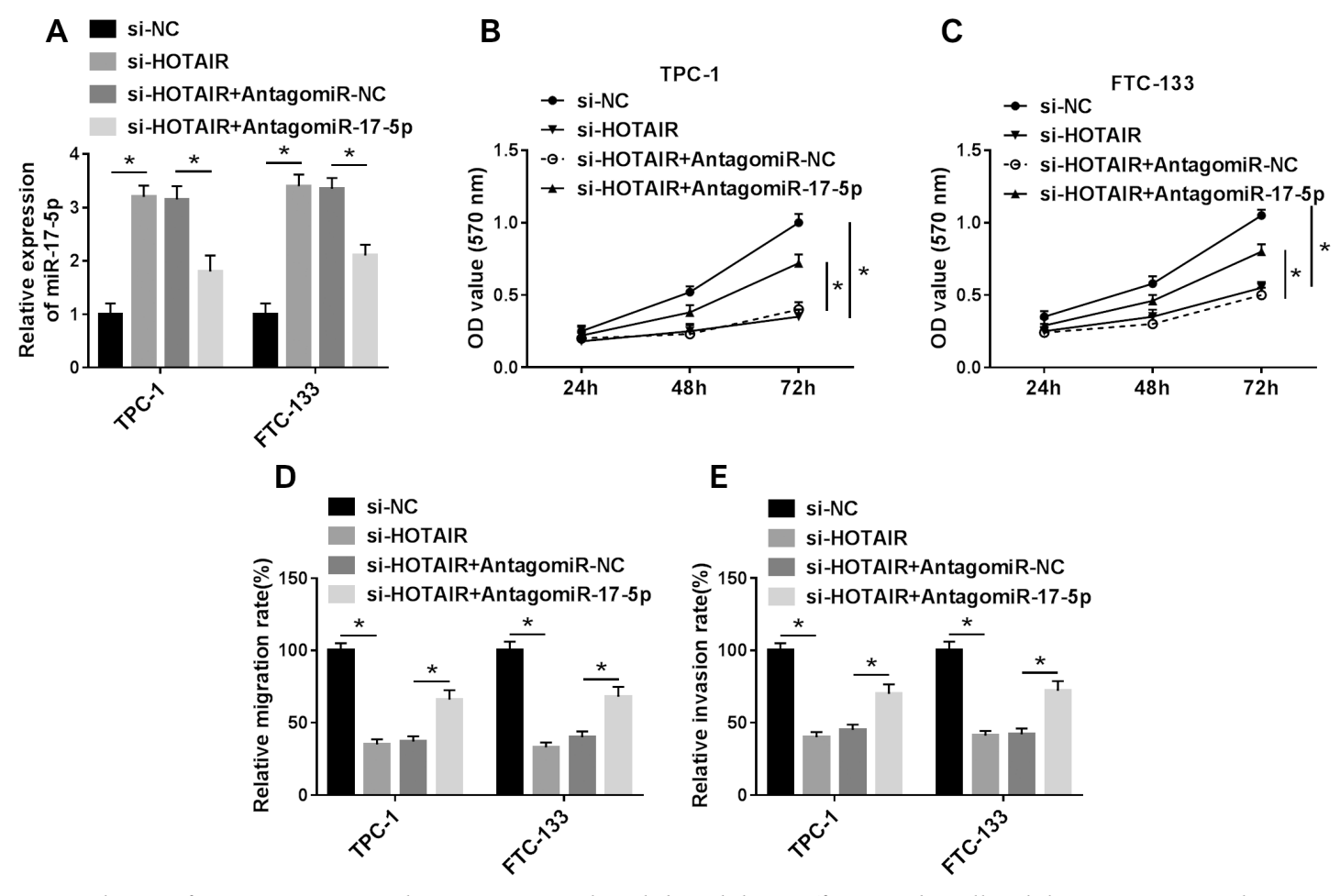

Figure 6. Downregulation of miR-17-5p reversed si-HOTAIR-mediated the inhibitor effects on the cell viability, migration and invasion abilities in thyroid cancer cells. TPC-1 and FTC-133 cells were transfected with si-NC, si-HOTAIR, si-HOTAIR + AntagomiR-NC or si-HOTAIR+AntagomiR17-5p. A) RT-qPCR was utilized to detect miR-17-5p expression in TPC-1 and FTC-133 cells after transfection. B and C) MTT assay for TPC-1 and FTC-133 cells after co-transfection for $24 \mathrm{~h}, 48 \mathrm{~h}$ and $72 \mathrm{~h}$. D) The relative migration rate of TPC-1 and FTC-133 cells after co-transfection conducted on Transwell. E) The relative invasion rate of TPC-1 and FTC-133 cells after co-transfection conducted on Transwell. ${ }^{\star}$ p $<0.05$ compared to cells transfected with si-NC. ${ }^{*} \mathrm{p}<0.05$ compared to cells co-transfected with si-HOTAIR + AntagomiR-NC. 


\section{Discussion}

Thyroid cancer consists of tumors with various histogenetic base and clinical behavior, ranging from indolent tumors with low mortality in most cases, to very aggressive malignancies, which is located at the base of the neck. [19]. Although the incidence of thyroid cancer is rising continually, the mortality from thyroid cancer has varied minimally during the past several decades $[1,3]$. One exigent thing physicians who treat thyroid cancers need to do, is to seek proper therapeutic approach for patients with clinical behavior [3].

LncRNAs play pivotal roles in cellular processes significant for normal development and physiology, and aberrant expression of non-coding RNA is, in essence, related to human disease, including cancer [20]. LncRNA HOTAIR was initially described by Howard Chang's group in 2004 [21]. Subsequently, the functional roles of HOTAIR in tumor biology have been largely elucidated. For example, HOTAIR is relevant to hepatitis $\mathrm{B}$ virus-induced liver carcinogenesis [22]. HOTAIR contributed to MPTP-induced Parkinson's disease (PD) by regulating LRRK2 expression, which helped to understand the molecular mechanisms in PD [23]. HOTAIR was upregulated in osteoarthritis (OA) cartilage tissues. It could directly bind to miR-17-5p and indirectly upregulated FUT2 level, thereby contributed to OA progression via $\mathrm{Wnt} / \beta$-catenin pathway [24]. HOTAIR promoted the epithelial-to-mesenchymal transition in gastric cancer by mediating the transformation of histone $\mathrm{H} 3$ lysine 27 acetylation to methylation [25]. The expression of HOTAIR was enhanced in pancreatic cancer (PC) tissues and PC PANC-1 and AsPC-1 cells with radiation stimulation, and knockdown of HOTAIR facilitated radiosensitivity of PC cells by regulating autophagy. This study supplied a novel insight to improve radiotherapy efficiency in PC [26]. HOTAIR epigenetically inhibited miR-122 expression via DNA methylation, resulting in activation of Cyclin G1 and elevation of tumorigenicity in HCC, which provide new insight into the mechanism of HOTAIR-mediated hepatocarcinogenesis via repressing miR-122 [27]. Additionally, HOTAIR expression was increased in chronic myeloid leukemia (CML) cell lines and samples, and could be used as a potential therapeutic target for advanced CML [28]. The results of the present study showed that HOTAIR was upregulated in thyroid cancer tissues and cell lines, and silencing HOTAIR inhibited cell viability, migration and invasion abilities in thyroid cancer cells in vitro and tumor formation in vivo, which were in accordance with previous studies [10,29].

A former data suggested that HOTAIR promoted the development and progression of thyroid cancer via repression of microRNA-1 and activation of CCND2 [29]. Here, we made efforts to explore other potential mechanism of HOTAIR on regulation of thyroid cancer progression. MiR-17-5p was identified as a target of HOTAIR, and was counter-regulated by HOTAIR. The target reaction was also
A

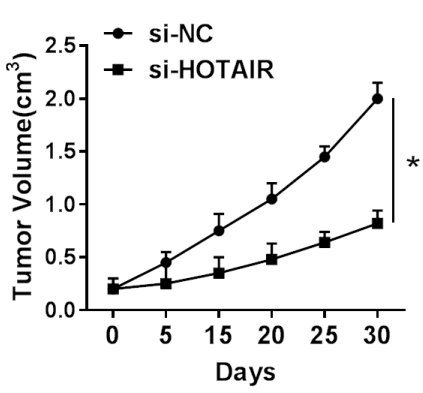

B

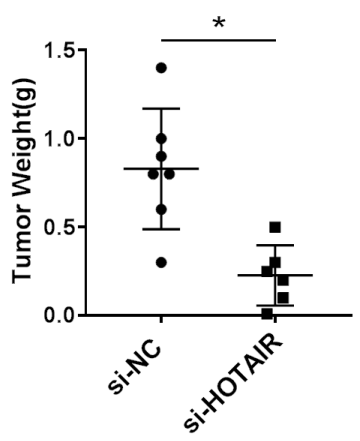

C

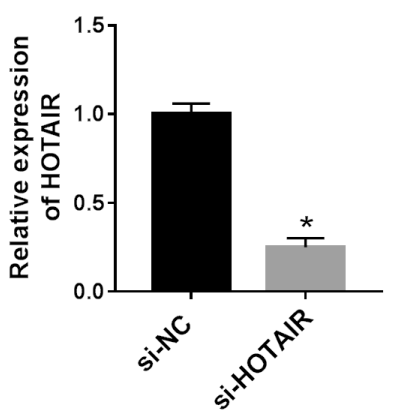

D

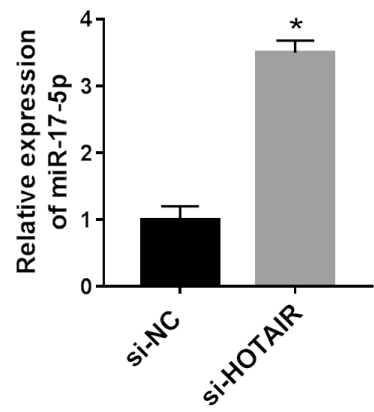

Figure 7. Knockdown of HOTAIR inhibited thyroid cancer tumorigenesis. Athymic mice were injected with FTC-133 cells transfected with siHOTAIR or si-NC. A) The tumor volume was measured at specific times. B) The tumor weight was measured at specific times. C) RT-qPCR assay for HOTAIR expression in athymic mice injected with FTC-133 cells transfected with si-HOTAIR or si-NC. D) RT-qPCR assay for miR-17-5p expression. ${ }^{*} p<0.05$ compared to mice injected with FTC-133 cells transfected with si-NC.

confirmed in studies of Wei et al. [7] and Hu et al. [24]. On the one hand, miR-17-5p serves as an oncogene, such as in osteosarcoma, miR-17-5p significantly upregulated in osteosarcoma tissues, linked with cell proliferation, invasion, advanced TNM stage and tumor growth [16]. Shan et al. pointed out that miR-17-5p promoted colorectal cancer tumorigenesis and progression via downregulating P130 and subsequently activated the Wnt/ $\beta$-catenin pathway [30]. A mature miR-17-5p can exert its facilitation on the hepatocellular carcinoma progression by repressing the expression of PTEN [31]. On the other hand, miR-17-5p functions as a tumor suppressor. In triple-negative breast cancer (TNBC), miR-17-5p inhibited TNBC cells proliferation, migration and invasion by targeting ETS variant 1 (ETV1), and miR-17-5p might be a therapeutic target for TNBC [32]. Mir-17-5p functions as a tumor suppressor in breast cancer cells by regulating oncogene AIB1 translation [33]. In addition, miR-17-5p was identified as a metastatic suppressor of basal-like breast cancer via a systematic analysis [34]. The current data clearly indicated that miR-17-5p was obviously downregulated in thyroid cancer tissues and cell lines. Gain of 
miR-17-5p blocked the cell viability, migration and invasion abilities in thyroid cancer cells and reversed si-HOTAIRmediated the repression effects on the cell viability, migration and invasion abilities in thyroid cancer cells.

In summary, we found that HOTAIR was significantly upregulated, while miR-17-5p was downregulated in thyroid cancer tissues and cell lines. In addition, both silencing HOTAIR and introduction of miR-17-5p efficiently inhibited the cell viability, migration and invasion abilities in thyroid cancer cells. Knockdown of HOTAIR also hindered thyroid cancer tumorigenesis in mice. MiR-17-5p was identified as a target of HOTAIR and was counter-regulated by HOTAIR. Rescue experiment indicated that gain of miR-17-5p reversed the silencing HOTAIR-induced the suppression effects on the cellular processes in thyroid cancer cells. In conclusion, HOTAIR promotes cell viability, migration and invasion in thyroid cancer by targeting miR-17-5p.

\section{References}

[1] COELHO RG, FORTUNATO RS, CARVALHO DP. Metabolic Reprogramming in Thyroid Carcinoma. Front Oncol 2018; 8: 82. https://doi.org/10.3389/fonc.2018.00082

[2] MORGANTI S, CEDA GP, SACCANI M, MILLI B, UGOLOTTI D et al. Thyroid disease in the elderly: sex-related differences in clinical expression. J Endocrinol Invest 2005; 28: 101-104.

[3] CABANillas ME, MCFADDEN DG, DURANTE C. Thyroid cancer. Lancet 2016; 388: 2783-2795. https://doi. org/10.1016/s0140-6736(16)30172-6

[4] CHOI C, THI THAO TRAN N, VAN NGU T, PARK SW, SONG MS et al. Promotion of tumor progression and cancer stemness by MUC15 in thyroid cancer via the GPCR/ERK and integrin-FAK signaling pathways. Oncogenesis 2018; 7: 85. https://doi.org/10.1038/s41389-018-0094-y

[5] LIU SJ, LIM DA. Modulating the expression of long noncoding RNAs for functional studies. EMBO Rep 2018; 19. https://doi.org/10.15252/embr.201846955

[6] JALALI S, BHARTIYA D, LALWANI MK, SIVASUBBU S, SCARIA V. Systematic transcriptome wide analysis of lncRNA-miRNA interactions. PLoS One 2013; 8: e53823. https://doi.org/10.1371/journal.pone.0053823

[7] WEI B, WEI W, ZHAO B, GUO X, LIU S. Long non-coding RNA HOTAIR inhibits miR-17-5p to regulate osteogenic differentiation and proliferation in non-traumatic osteonecrosis of femoral head. PLoS One 2017; 12: e0169097.https:// doi.org/10.1371/journal.pone.0169097

[8] SVOBODA M, SLYSKOVA J, SCHNEIDEROVA M, MAKOVICKY P, BIELIK L et al. HOTAIR long non-coding RNA is a negative prognostic factor not only in primary tumors, but also in the blood of colorectal cancer patients. Carcinogenesis 2014; 35: 1510-1515. https://doi.org/10.1093/carcin/bgu055

[9] ZHU H, LV Z, AN C, SHI M, PAN W et al. Onco-lncRNA HOTAIR and its functional genetic variants in papillary thyroid carcinoma. Sci Rep 2016; 6: 31969. https://doi. org/10.1038/srep31969
[10] ZHANG Y, YU S, JIANG L, WANG X, SONG X. HOTAIR is a promising novel biomarker in patients with thyroid cancer. Exp Ther Med 2017; 13: 2274-2278. https://doi.org/10.3892/ etm.2017.4231

[11] BOUFRAQECH M, KLUBO-GWIEZDZINSKA J, KEBEBEW E. MicroRNAs in the thyroid. Best Pract Res Clin Endocrinol Metab 2016; 30: 603-619. https://doi.org/10.1016/j. beem.2016.10.001

[12] AMBROS V. microRNAs: tiny regulators with great potential. Cell 2001; 107: 823-826. https://doi.org/10.1016/s00928674(01)00616-X

[13] LIMA CR, GOMES CC, SANTOS MF. Role of microRNAs in endocrine cancer metastasis. Mol Cell Endocrinol 2017; 456: 62-75. https://doi.org/10.1016/j.mce.2017.03.015

[14] FORTE S, LA ROSA C, PECCE V, ROSIGNOLO F, MEMEO L. The role of microRNAs in thyroid carcinomas. Anticancer Res 2015; 35: 2037-2047.

[15] OLIVE V, LI Q, HE L. mir-17-92: a polycistronic oncomir with pleiotropic functions. Immunol Rev 2013; 253: 158166. https://doi.org/10.1111/imr.12054

[16] WANG W, ZHANG L, ZHENG K, ZHANG X. miR-17-5p promotes the growth of osteosarcoma in a BRCC2-dependent mechanism. Oncol Rep 2016; 35: 1473-1482. https:// doi.org/10.3892/or.2016.4542

[17] LIU L, YANG J, ZHU X, LI D, LV Z et al. Long noncoding RNA H19 competitively binds miR-17-5p to regulate YES1 expression in thyroid cancer. FABS J 2016; 283: 2326-2339. https://doi.org/10.1111/febs.13741

[18] SAITO K, OKU T, ATA N, MIYASHIRO H, HATTORI M et al. A modified and convenient method for assessing tumor cell invasion and migration and its application to screening for inhibitors. Biol Pharm Bull 1997; 20: 345-348. https:// doi.org/10.1248/bpb.20.345

[19] TASOULAS J, TSOUROUFLIS G, THEOCHARIS S. Neovascularization: an attractive but tricky target in thyroid cancer. Expert Opin Ther Targets 2018; 22: 799-810. https://doi. org/10.1080/14728222.2018.1513494

[20] LORENZI L, AVILA COBOS F, DECOCK A, EVERAERT $\mathrm{C}$, HELSMOORTEL $\mathrm{H}$ et al. Long non-coding RNA expression profiling in cancer: challenges and opportunities. Genes Chromosomes Cancer 2019; 58: 191-199. https://doi. org/10.1002/gcc.22709

[21] MATTICK JS. RNA regulation: a new genetics? Nat Rev Genet 2004; 5: 316-323. https://doi.org/10.1038/nrg1321

[22] ZHANG H, DIAB A, FAN H, MANI SK, HULLINGER R et al. PLK1 and HOTAIR Accelerate Proteasomal Degradation of SUZ12 and ZNF198 during Hepatitis B Virus-Induced Liver Carcinogenesis. Cancer Res 2015; 75: 2363-2374. https://doi.org/10.1158/0008-5472.can-14-2928

[23] LIU S, CUI B, DAI ZX, SHI PK, WANG ZH et al. Long Non-coding RNA HOTAIR Promotes Parkinson's Disease Induced by MPTP Through up-regulating the Expression of LRRK2. Curr Neurovasc Res 2016; 13: 115-120. https://doi. org/10.2174/1567202613666160316155228

[24] HU J, WANG Z, SHAN Y, PAN Y, MA J et al. Long noncoding RNA HOTAIR promotes osteoarthritis progression via miR-17-5p/FUT2/beta-catenin axis. Cell Death Dis 2018; 9: 711. https://doi.org/10.1038/s41419-018-0746-Z 
[25] SONG Y, WANG R, LI LW, LIU X, WANG YF et al. Long non-coding RNA HOTAIR mediates the switching of histone $\mathrm{H} 3$ lysine 27 acetylation to methylation to promote epithelial-to-mesenchymal transition in gastric cancer. Int J Oncol 2019; 54: 77-86. https://doi.org/10.3892/ijo.2018.4625

[26] WU C, YANG L, QI X, WANG T, LI M et al. Inhibition of long non-coding RNA HOTAIR enhances radiosensitivity via regulating autophagy in pancreatic cancer. Cancer Manag Res 2018; 10: 5261-5271. https://doi.org/10.2147/cmar. s174066

[27] CHENG D, DENG J, ZHANG B, HE X, MENG Z et al. LncRNA HOTAIR epigenetically suppresses miR-122 expression in hepatocellular carcinoma via DNA methylation. EBioMedicine 2018; 36: 159-170. https://doi.org/10.1016/j. ebiom.2018.08.055

[28] LI Z, LUO J. Epigenetic regulation of HOTAIR in advanced chronic myeloid leukemia. Cancer Manag Res 2018; 10: 5349-5362. https://doi.org/10.2147/cmar.s166859

[29] DI W, LI Q, SHEN W, GUO H, ZHAO S. The long non-coding RNA HOTAIR promotes thyroid cancer cell growth, invasion and migration through the miR-1-CCND2 axis. Am J Cancer Res 2017; 7: 1298-1309.
[30] MA Y, ZHANG P, WANG F, ZHANG H, YANG Y et al. Elevated oncofoetal miR-17-5p expression regulates colorectal cancer progression by repressing its target gene P130. Nat Commun 2012; 3: 1291. https://doi.org/10.1038/ncomms2276

[31] SHAN SW, FANG L, SHATSEVA T, RUTNAM ZJ, YANG X et al. Mature miR-17-5p and passenger miR-17-3p induce hepatocellular carcinoma by targeting PTEN, GalNT7 and vimentin in different signal pathways. J Cell Sci 2013; 126: 1517-1530. https://doi.org/10.1242/jcs.122895

[32] LI J, LAI Y, MA J, LIU Y, BI J et al. miR-17-5p suppresses cell proliferation and invasion by targeting ETV1 in triplenegative breast cancer. BMC Cancer 2017; 17: 745. https:// doi.org/10.1186/s12885-017-3674-x

[33] HOSSAIN A, KUO MT, SAUNDERS GF. Mir-17-5p regulates breast cancer cell proliferation by inhibiting translation of AIB1 mRNA. Mol Cell Biol 2006; 26: 8191-8201. https:// doi.org/10.1128/mcb.00242-06

[34] FAN M, SETHURAMAN A, BROWN M, SUN W, PFEFFER LM. Systematic analysis of metastasis-associated genes identifies miR-17-5p as a metastatic suppressor of basal-like breast cancer. Breast Cancer Res Treat 2014; 146: 487-502. https://doi.org/10.1007/s10549-014-3040-5 